\title{
Die Euro-Verfassung und die Ewigkeitsklausel in Karlsruhe, Teil II: Stabilisierungsmechanismus
}

VB verfassungsblog.de/die-euroverfassung-und-die-ewigkeitsklausel-karlsruhe-teil-stabilisierungsmechanismus/

Christoph Möllers So 22 Jul 2012

So 22 Jul

2012

Leider hat es etwas länger mit Teil II gedauert. In der Zwischenzeit ist der Autor, gemeinsam mit Martin Nettesheim aus Tübingen, zum Prozessvertreter des Deutschen Bundestages im Verfahren bestellt worden. Er muss sich also um Objektivität bemühen, die Leser seien in jedem Fall zu besonders misstrauischem Lesen ermuntert.

Der Europäische Stabilisierungsmechanismus (ESM) dürfte den Befürchtungen des Zweiten Senats aus zwei Gründen zumindest näher kommen als der Fiskalvertrag: zum Ersten, weil es in inm um die Verteilung immenser Mittel geht, welche die Handlungsfähigkeit des Bundes wesentlich einschränken könnte - eben nicht wie im Fiskalvertrag um deren Einsparung, die zumindest langfristig neue Handungspielräume ermöglichen mag. Zum Zweiten, weil diese Verteilung von einer veritablen neuen internationalen Organisation vorgenommen wird, die eigene Entscheidungsspielräume erhält. Der Vertrag ist kompliziert, an dieser Stelle muss stark vereinfacht werden. Dass er einen verbotenen Haftungsautomatismus vorsieht, durch den der Bundesrepublik allein auf Grundlage der Entscheidung anderer Staaten undefinierte Zahlungsverpflichtungen erwachsen könnten, ist kaum zu erkennen. Dass das System aber im Ganzen einer politischen Logik folgt, die für die Bundesrepublik allein schwer zu beherrschen ist, erscheint gleichfalls schwer zu bezweifeln. Bei einer Folgenabwägung wird aber auch zu bedenken sein, was ein Ausstieg aus diesem System bedeuten würde und ob dessen Folgen besser zu überschauen sind.

Konkreter sind es wohl drei Grundsätze, denen die Organisation des ganzen Mechanismus aus der Sicht des Zweiten Senats wird folgen müssen: Zum Ersten müssen die zu verteilenden Mittel begrenzt sein. Das heisst, die für den ESM konstitutiven Regeln haben einen Betrag zu nennen, bei dem die Haftung endet. Zum Zweiten müssen alle relevanten Entscheidungen des Mechanismus mit Zustimmung der Bundesrepublik ergehen oder umgekehrt: diese muss ein Veto-Recht haben. Zum Dritten muss der Bundestag wichtigen haushaltsrelevanten Entscheidungen zustimmen.

Hinsichtlich des ersten Punktes scheint der ESM-Vertrag jedenfalls der Papierform nach keine Probleme zu bereiten. Er formuliert eine absolute Haftungsgrenze, die "unter allen Umständen" gilt. Ob sich eine solche Regelung umgehen lässt, erscheint zweifelhaft. Solche Szenarien spielen allerdings im Verfahren eine gewisse Rolle. Könnte sich aus Nachschusspflichten, aus der Ausgabe von Kapital abweichend vom Nennwert oder aus Abmachungen der anderen Parteien nicht doch eine höhere Haftung ergeben? Auch wenn solche Szenarien konstruiert wirken, verdienen sie eine genaue Kontrolle durch das Gericht. Schließlich haben andere Vertragsstaaten nicht notwendig dieselben Interessen wie die Bundesrepublik.

Auch der zweite Punkt scheint im ESM vergleichsweise solide abgesichert zu sein. In den Gremien des ESM werden alle wesentlichen Entscheidungen entweder einstimmig oder mit einer qualifizierten Mehrheit beschlossen. Für letztere verfügt die Bundesrepublik dank ihres Kapitalanteils über eine Veto-Position. Dies könnte wohl nur durch einen Beitritt Großbritanniens geändert werden, dem der Bund seinerseits zustimmen müsste - keine besonders wahrscheinliche Variante, zudem eine, die die Bundesrepublik finanziell entlasten würde. Auch hier bedarf es einer gewissen Phantasie, um ein Problem zu sehen: Was passiert etwa, wenn der deutsche Vertreter nicht anwesend ist oder nicht die Interessen vertritt, die der Bundestag definiert hat? Wiederum könnte das Bundesverfassungsgericht nach der Möglichkeit härterer Sicherungsmechanismen suchen. Auch die Frage, wer von der Bundesrepublik in das eigentlich operative Gremium, das Direktorium, geschickt wird, mag eine Rolle spielt. Die Bundesregierung will einen Staatssekretär, also einen weisungsabhängigen Beamten entsenden.

Verfassungsrechtlich schwieriger sähe es aus, wenn eine Privatperson allein aufgrund ihrer wirtschaftlichen Kompetenz im Gremium säße. 
Für den dritten Punkt stellt sich die Frage, was denn nun relevante Entscheidungen sind, die der Bundestag selbst zu treffen hat. Immerhin liegt zu dieser Frage bereits eine Entscheidung des Senats zum EFSF vom 7. September 2011 vor. Diese spielte bei der Ausarbeitung des Begleitgesetzes eine wichtige Rolle. Ohne Anspruch auf Vollständigkeit lassen sich einige Entscheidungskomplexe benennen: die Entscheidung über die Höhe der eingesetzten Mittel, die Frage, für wen sie eingesetzt werden, welchen Finanzierungsinstrumente anzuwenden sind und unter welchen Bedingungen dies geschieht. Tatsächlich hat der Bundestag in all den genannten Fragen ein Entscheidungsrecht, er muss auch den Bedingungen zustimmen, unter denen Mittel an einen notleidenden Staat vergeben werden. Aber auch hier sind Szenarien denkbar, in denen der Bundestag außen vor bliebe. Etwa wenn der Bund für Verluste aufkommen muss, die der ESM in seinen Anlagen erleidet. Schließlich bleibt die Frage, inwieweit es eine verfassungsrechtliche Pflicht geben könnte, die im Vertrag vorgesehene Verschwiegenheitspflicht gegenüber dem Bundestag und der demokratischen Öffentlichkeit zu beschränken.

Für die Abgeordneten bleibt in jedem Fall viel, vielleicht mitunter sogar zu viel zu entscheiden. Vergessen wir zweierlich nicht, was in der Diskussion im Moment zu sehr in den Hintergrund gerät: In einer parlamentarischen Demokratie handelt die Regierung stets unter der Bedingung des politischen Vertrauens der parlamentarischen Mehrheit. Das Parlament steht nicht einfach gegen die Regierung. Und: In einem gewaltengegliederten System ist es gerade kein verfassungsrechtlicher Idealzustand, wenn jede Entscheidung vom Parlament gefasst wird. Weil es sich um so große Summen handelt, erscheint es angemessen, die parlamentarische Beteiligung zu intensivieren. Es ist richtig, dass der Bundestag letzte Woche über die Spanienhilfe entscheiden musste. Aber auch diese Beteiligung stößt an Grenzen organisierbarer Arbeitsteilung.

Am 12. September wissen wir mehr.

LICENSED UNDER CC BY NC ND

SUGGESTED CITATION Möllers, Christoph: Die Euro-Verfassung und die Ewigkeitsklausel in Karlsruhe, Teil II: Stabilisierungsmechanismus, VerfBlog, 2012/7/22, http://verfassungsblog.de/die-euroverfassung-und-dieewigkeitsklausel-karlsruhe-teil-stabilisierungsmechanismus/. 